\title{
Validación de una escala para medir afrontamiento ante ciberagresiones entre universitarios
}

\section{Validation of a Scale to Measure Coping with Cyber-Bullying in University Students}

\author{
Gisela Margarita Torres-Acuña (1) gisela.torres@itson.edu.mx \\ Claudia Rivera Hernández (2) claudia.rivera@correo.buap.mx \\ Yadira Navarro Rangel (2) yadira.navarro@correo.buap.mx \\ (1) Instituto Tecnológico de Sonora \\ (2) Benemérita Universidad Autónoma de Puebla \\ (Recibido: 30 de marzo de 2017; Aceptado para su publicación: 21 de junio de 2017)
}

Cómo citar: Torres-Acuña, G. M., Rivera, C. y Navarro, Y. (2019). Validación de una escala para medir afrontamiento ante ciberagresiones entre universitarios. Revista Electrónica de Investigación Educativa, 21, e09, 1-11.

doi:10.24320/redie.2019.21.e09.1907

\section{Resumen}

En el estudio se analizaron las propiedades psicométricas de una escala para medir afrontamiento de estudiantes universitarios a ciberagresiones. Se seleccionó de forma no probabilística a 346 estudiantes de educación superior, $85.8 \%$ mujeres ( $M=20.74$, $D E=3.33$ años) y $14.2 \%$ hombres $(M=20.84, D E=2.30$ años) de una universidad pública del sur de Sonora (México). Se llevó a cabo el análisis factorial exploratorio y confirmatorio, así como de fiabilidad con los coeficientes de Alfa de Cronbach, Omega de McDonald y el análisis de la varianza media extractada. Los resultados arrojaron un modelo de medida sustentable del afrontamiento a la ciberagresión integrado por tres factores: búsqueda de apoyo social, solución técnica evasiva y solución técnica proactiva. Se concluye que la escala cuenta con las propiedades psicométricas necesarias para su empleo en la medición del constructo entre estudiantes universitarios.

Palabras clave: Acoso cibernético, propiedades psicométricas, educación superior.

\section{Abstract}

This study analyzed the psychometric properties of a scale to measure coping with cyber-bullying among university students. Non-probability sampling was used to select 346 higher education students from a public university in southern Sonora (Mexico). Exploratory and confirmatory factor analysis was performed, reliability was tested with Cronbach's alpha and McDonald's omega, and the mean variance was analyzed. The results revealed a sustainable model for measuring coping with cyber-bullying that comprised three factors: seeking social support, evasive technical solution, and proactive technical solution. It is concluded that the scale has the necessary psychometric properties to measure this construct in university students. 


\section{Introducción}

El ciberbullying constituye un fenómeno de interés alrededor del mundo debido a su prevalencia entre estudiantes de distintos niveles educativos y los efectos que alcanza sobre su desarrollo socioemocional (Chapell et al., 2006; Garaigordobil, 2011; Perren, Dooley, Shaw, y Cross, 2010; Sahin, Aydin y Sari, 2012).

En la literatura especializada se define al ciberbullying como una modalidad del bullying tradicional, que consiste en el empleo de medios tecnológicos para causar daño a otro u otros de forma intencional y repetitiva (Belsey, 2005; Li, 2008; Olweus, 2012; Tokunaga, 2010); y la cual genera mayor impacto en la víctima (Parris, Varjas, Meyers y Cutts, 2012) debido a: a) el posible anonimato del agresor, b) el número de espectadores alcanzados y c) la permanencia de la ciberagresión por tiempo indefinido (Buelga, Cava, y Musitu, 2010; García-Maldonado, Joffre-Velázquez, Martínez-Salazar, y Llanes-Castillo, 2011; Nocentini et al., 2010; Slonje y Smith, 2008).

La prevalencia de este tipo de agresión entre los jóvenes se reconoce en diversos niveles educativos en el contexto nacional e internacional, desde un 11\% hasta un $60 \%$ en las poblaciones estudiadas (Hoff y Mitchell, 2009; Holfeld y Leadbeater, 2014; Puc y Rodríguez, 2015; Valdés, Carlos, Tánori y Wendlandt, 2014; Varela, Pérez, Schwaderer, Astudillo y Lecannelier, 2014; Vega-López, González-Pérez, y QuinteroVega, 2013), empleando principalmente el teléfono móvil y las herramientas de Internet (Buelga et al., 2010; Oliveros et al., 2012).

Ante este contexto, analizar los factores relacionados a la prevalencia del ciberbullying cobra importancia, ya que permite orientar acciones de prevención. Uno de las variables estudiadas y con evidencia empírica de su relación con la victimización es el afrontamiento que los estudiantes llevan a cabo como respuesta a la agresión (Kristensen y Smith, 2003). Machackova, Cerna, Sevcikova, Dedkova y Danebach (2013) al examinar qué tipo de afrontamiento llevan a cabo las cibervíctimas de agresión entre estudiantes, y su contribución en la disminución de las ciberagresiones, encuentran que las más efectivas son aquellas enfocadas a la solución del problema, a través de estrategias de solución tecnológica y búsqueda de soporte social, más que la confrontación y represalias. Por otro lado, el afrontamiento pasivo o emocional suelen caracterizar a los estudiantes cibervictimizados (Moral, López, Díaz y Cienfuegos, 2011; TorresAcuña, Rivera, Navarro y Sandoval, 2016).

Este tipo de resultados contribuye a la idea de que el afrontamiento que una persona utiliza puede atraer efectos positivos o negativos, influir en la posibilidad de vivir la situación como estresante o suprimir la sensación de estrés (Fernández y Díaz, 2001; Lazarus y Folkman, 1986; Paul, Smith, y Blumberg, 2012).

\subsection{Referente teórico}

Afrontamiento es un término acuñado a la Teoría del Estrés (Lazarus y Folkman, 1984) que destaca la interacción entre la persona y el ambiente -donde el estrés es concebido como un desequilibrio entre la percepción sobre las demandas que se imponen y la percepción de los recursos que se tienen para sobrellevar dichas demandas, evaluadas como amenazantes o desbordante de dichos recursos (Canessa, 2002). Así, el afrontamiento se define como aquellos esfuerzos que realiza una persona para tratar situaciones que le causan malestar, y que superan sus propios recursos (Lazarus y Folkman, 1984), incluso Frydenberg y Lewis (1994) señalan que el afrontamiento puede ser apreciado como una competencia psicosocial que incluye estrategias conductuales y cognitivas utilizadas para enfrentar las demandas de la vida.

Lazarus y Folkman (1984) se refieren al afrontamiento como un proceso que incluye dos niveles de evaluación, determinados por las creencias y expectativas sobre el mundo y los recursos con que se cuenta. En un primer momento, el individuo valora si está o no frente a una situación estresante o de peligro, y posteriormente estima la forma en que puede reaccionar frente a dicho suceso considerando los posibles resultados. 
Los enfoques del afrontamiento definidos por Lazarus y Folkman (1987) son dos: el centrado en el problema, que busca el desarrollo de acciones que permitan cambiar la relación de la persona y su entorno; y el centrado en la emoción, en donde se busca regular la angustia emocional. La utilización de éstos depende del grado de control que se tenga sobre la situación (Canessa, 2002; Perren et al., 2012).

La importancia del afrontamiento que un individuo lleva a cabo radica en el supuesto de que éste puede influir en el nivel de angustia que se experimenta en situaciones de intimidación (Paul et al., 2012); si el afrontamiento es ineficaz, las consecuencias para la persona se ven reflejadas en su salud, moral y funcionamiento social; en cambio, si es eficaz el estrés se mantiene bajo control sin existir daño físico o psicológico significativo (Lazarus, 2006).

Frente al fenómeno del ciberbullying, la literatura reconoce como estrategias de afrontamiento útiles para las víctimas: la búsqueda de apoyo social (Causey y Dubow, 1992; Lin, Dean y Ensel, 1981; Perren et al., 2012) y la solución técnica (Machackova et al., 2013; Perren et al., 2012; Price y Dalgleish, 2010).

La búsqueda de apoyo social implica compartir el problema con otras personas con la finalidad de obtener ayuda para su resolución (Frydenberg y Lewis, 1999). Este afrontamiento es muy empleado en experiencias de bullying y ciberbullying, situación que resulta coherente considerando la existencia de un desequilibrio de poder entre agresor y víctima, lo cual sugiere que sin ayuda la víctima no podría manejar la situación (Sevcíkova, Machackova, Wright, Debková y Cerna, 2015). En este sentido, estudiantes con experiencias de ciberbullying manifiestan el mayor uso del afrontamiento de búsqueda de ayuda, lo cual además-consideran de utilidad (Machackova et al., 2013; Paul et al., 2012).

Por otro lado, la solución técnica o tecnológica refiere acciones específicas sobre el manejo de herramientas tecnológicas, por ejemplo, bloquear al agresor o borrar el mensaje que le agrede (Aricak et al., 2008; Slonje, Smith y Frisén, 2013; Tokunaga, 2010). Dada la naturaleza del ciberbullying, que demanda el empleo de tecnología para las ciberagresiones, el aspecto técnico cobra relevancia. Algunos estudios señalan que su empleo es comúnmente utilizado por los jóvenes ante la ciberagresión, con acciones como: bloquear mensajes o identidades, cambiar cuentas de correo electrónico o números de teléfono, y así detener la comunicación con el agresor (Price y Dalgleish, 2010; Slegová y Cerna, 2011; Smith et al., 2008; Tokunaga, 2010). Ante este contexto, se reconoce la importancia de este afrontamiento como factor inhibidor de los efectos del ciberbullying en las víctimas (Machmutow, Perren, Sticca, y Alsaker, 2012), y sobre todo de su medición.

A pesar de que existen diversos instrumentos para medir el afrontamiento, se han analizado algunas implicaciones importantes para creer que es necesario el desarrollo de escalas para la medición del constructo (Nava, Ollua, Vega, y Soria, 2010), especialmente frente al ciberbullying. Algunas reflexiones son: a) la mayoría de los estudios retoman escalas para la medición del afrontamiento al estrés que luego es relacionado con la prevalencia de ciberagresiones entre estudiantes, pero muy pocos se centran en la medición del afrontamiento frente a situaciones específicas de ciberagresión (Machackova et al., 2013; Völlink, Bolman, Dehue, y Jacobs, 2013; Wachs, Wolf, y Pan, 2012); b) se han empleado instrumentos que miden el afrontamiento al ciberbullying, sin embargo, no reportan evidencias de validez y fiabilidad (Aricak et al., 2008; Machackova et al., 2013); c) existen pocos instrumentos que miden el afrontamiento al ciberbullying con población universitaria (Schenk y Fremouw, 2012) -en especial en México; y d) la mayoría de las escalas se encuentran en idioma inglés y son escasos los elaborados o adaptados al español (Nacimiento y Mora-Merchán, 2014). Ante esta situación, el estudio realizado busca determinar las propiedades psicométricas (fiabilidad y validez de constructo) de una escala para medir el afrontamiento a ciberagresiones en estudiantes mexicanos de educación superior.

\section{Método}

A través de un muestreo no probabilístico se seleccionaron 346 estudiantes de la carrera de Educación inscritos durante el período de agosto-diciembre de 2016 en una institución de educación superior del sur de Sonora: 297 mujeres (85.8\%) con una edad promedio de 20.74 (DE=3.33 años) y 49 hombres (14.2\%) con una edad promedio de 20.84 ( $D E=2.3$ años). En el momento de la recolección de datos 89 estudiantes 
(25.7\%) cursaban el primer semestre, 82 (23.7\%) el tercer semestre, 103 (29.8\%) el quinto semestre y 72 (20.8\%) el séptimo. Para llevar a cabo el análisis factorial exploratorio y confirmatorio la muestra se dividió aleatoriamente en: submuestra 1 (SM1) con 173 (50\%) estudiantes y submuestra 2 (SM2) con 173 (50\%) estudiantes.

Para desarrollar el instrumento "Afrontamiento ante Ciberagresiones en Educación Superior" (ACES) se retomó la dimensión de Apoyo social de la adaptación del Inventario de Estrategias de Afrontamiento (CSI, por sus siglas en inglés) al ámbito español realizado por Cano, Rodríguez y García (2007), y los aportes de Livingstone, Haddon, Görzig y ólafsson (2011) para medir Solución técnica.

La escala se compone de 13 ítems que miden el afrontamiento ante las ciberagresiones, agrupados en dos dimensiones: 1) Búsqueda de apoyo social, mediante cinco ítems mide acciones de búsqueda de soporte emocional (por ejemplo: "Pido consejo a una persona de mi respeto"), y 2) Solución técnica, con ocho ítems evalúa acciones de afrontamiento en el ciberespacio (por ejemplo: "Bloqueo al agresor que entra en contacto conmigo"). El cuestionario tiene un formato tipo Likert de opciones que van desde Nunca (0 veces) hasta Siempre (más de 10 veces) en los últimos seis meses.

Para el levantamiento de datos se solicitó permiso a las autoridades académicas y profesores, se explicó el objetivo del estudio y se aseguró la confidencialidad de la información proporcionada por cada participante. Posteriormente, se invitó a los estudiantes a participar y se les explicó el objetivo del estudio; asimismo, se les informó sobre el anonimato y confidencialidad de la información proporcionada, los casos que accedieron firmaron una carta de consentimiento informado.

El análisis de los datos se llevó a cabo en varios momentos: primero, se realizó un análisis descriptivo de los datos con el fin de verificar la distribución de los puntajes; segundo, se retomó la SM1 para llevar a cabo el análisis factorial exploratorio con el método de extracción de máxima verosimilitud y rotación Oblimin directo, para determinar la estructura factorial de los ítems y el porcentaje de varianza explicada. Para ello, se revisó el valor del índice de Kaiser-Meyer-Olkin (KMO) y la prueba de Bartlett para verificar la pertinencia del análisis según el tamaño de la muestra; así como la comunalidad de cada ítem, donde se decidió omitir aquellos con valor por debajo de .30, y con carga factorial menor a 30 en un factor o mayor en dos o más factores (Cea, 2004; De Vellis, 2012), para esto se empleó el programa sPSs 23.

Por último, con la SM2 y empleando la estructura factorial previamente hallada, se realizó un análisis factorial confirmatorio utilizando el método de ecuaciones estructurales. Se empleó el método bootstrap (con 500 repeticiones con un intervalo de confianza del 90\%) con el fin de no afectar los resultados por problemas de normalidad (Brown, 2015; Byrne, 2010). En el análisis de bondad de ajuste del modelo propuesto se utilizó el método de máxima verosimilitud (ML) y los índices de ajuste propuestos por Brown (2015) y Byrne (2010): $X^{2}$, p (chi-cuadrada y probabilidad asociada), SRMR (raíz cuadrada residual estandarizada), AGFI (índice de bondad de ajuste ajustado), CFI (índice de ajuste comparativo), RMSEA IC 90 (error de la raíz cuadrada de la media de aproximación con su intervalo de confianza). Todo esto con apoyo del Amos 23. Para determinar la fiabilidad de la escala se emplearon los métodos de consistencia interna a través del coeficiente Alfa de Cronbach, Omega de McDonald $(\Omega)$ y el análisis de la Varianza Media Extractada (VME).

\section{Resultados}

\subsection{Análisis descriptivo}

Con el objetivo de revisar la distribución de los datos se llevó a cabo un análisis descriptivo con el que se reconocen ligeras desviaciones de normalidad en seis ítems (ejemplo: "Elimino mi cuenta" [red social, correo electrónico, etc.]) (ver tabla I), lo que debe ser considerado en análisis posteriores para no afectar los resultados -como el hecho de emplear el método bootstrap en el análisis factorial confirmatorio. 
Tabla I. Media, desviación estándar, asimetría y curtosis de los ítems

\begin{tabular}{l|r|c|c|c}
\hline \multicolumn{1}{c|}{ Ítems } & M & DE & Asimetría & Curtosis \\
\hline Cuento con alguien que escucha mi problema & 2.78 & 1.43 & -.83 & -.72 \\
\hline Dejo que mis amigos me echen la mano & 2.34 & 1.31 & -.30 & -.98 \\
Hablo con una persona de confianza sobre mi problema & 2.90 & 1.38 & -.98 & -.39 \\
\hline Paso tiempo con mis amigos & 2.65 & 1.34 & -.64 & -.80 \\
\hline Pido consejo a una persona de mi respeto & 2.62 & 1.35 & -.58 & -.90 \\
\hline Bloqueo al agresor que entra en contacto conmigo & 1.94 & 1.65 & .06 & -1.64 \\
Borro el mensaje que me daña & 2.37 & 1.59 & -.34 & -1.47 \\
\hline Borro o pido al administrador del sitio web que borre la publicación que me daña & 1.86 & 1.64 & .11 & -1.60 \\
Cambio contraseña, nombre de usuario, cuenta de correo & 1.49 & 1.53 & .56 & -1.16 \\
\hline Cambio mi número de teléfono & .84 & 1.29 & 1.32 & .41 \\
Dejo de usar la aplicación o tecnología por medio de la cual me perjudican & 1.04 & 1.35 & 1.02 & -.31 \\
\hline Elimino mensajes anónimos sin leerlos & 1.37 & 1.49 & .62 & -1.08 \\
Elimino mi cuenta (red social, correo electrónico, etc.) & .59 & 1.11 & 1.86 & 2.34 \\
\hline
\end{tabular}

\subsection{Validez de constructo}

Análisis factorial exploratorio. Los resultados del índice de KMO (0.85) y la prueba de Bartlett $\left(X^{2}=2189.61, p<.000\right)$ para la SM1 sugirieron que los datos son adecuados para este análisis. Al revisar las comunalidades de los 13 ítems originales se identificó uno con valor menor a .30 (Elimino mensajes anónimos sin leerlos) lo que significa que tiene poca relación con el resto, por lo que se eliminó.

La propuesta original consideró una escala de 13 ítems agrupados en dos dimensiones (Búsqueda de apoyo social y Solución técnica). Sin embargo, como resultado del análisis factorial exploratorio se obtuvo una escala de 12 ítems agrupados en 3 dimensiones que explican el 60.52\% de la varianza total del constructo (ver tabla II). La dimensión Búsqueda de apoyo social se mantuvo como la original (5 ítems, $34.77 \%$ de la varianza), mientras que la dimensión Solución técnica se dividió en 2, las cuales se renombraron como: Solución técnica evasiva (4 ítems, 19.95\% de la varianza), por tratarse de acciones donde el estudiante deja de usar la tecnología por medio de la cual recibe la ciberagresión y Solución técnica proactiva (3 ítems, 5.80\% de la varianza) referida a acciones para eliminar u omitir la ciberagresión recibida. 
Tabla II. Resultado del análisis factorial exploratorio con tres factores

\begin{tabular}{|c|c|c|c|c|}
\hline \multirow{2}{*}{ Ítems } & \multicolumn{3}{|c|}{ Carga } & \multirow[b]{2}{*}{$\mathrm{h}^{2}$} \\
\hline & $\mathrm{F} 1$ & $\mathrm{~F} 2$ & F3 & \\
\hline Cuento con alguien que escucha mi problema & .89 & & & .77 \\
\hline Dejo que mis amigos me echen la mano & .73 & & & .57 \\
\hline Hablo con una persona de confianza sobre mi problema & .94 & & & .84 \\
\hline Paso tiempo con mis amigos & .72 & & & .52 \\
\hline Pido consejo a una persona de mi respeto & .72 & & & .59 \\
\hline Cambio contraseña, nombre de usuario, cuenta de correo & & .57 & & .56 \\
\hline Cambio mi número de teléfono & & .77 & & .61 \\
\hline Dejo de usar la aplicación o tecnología por medio de la cual me perjudican & & .66 & & .52 \\
\hline Elimino mi cuenta (red social, correo electrónico, etc.) & & .86 & & .65 \\
\hline Bloqueo al agresor que entra en contacto conmigo & & & .66 & .45 \\
\hline Borro el mensaje que me daña & & & .81 & .64 \\
\hline Borro o pido al administrador del sitio web que borre la publicación que me daña & & & .65 & .56 \\
\hline \multicolumn{5}{|l|}{ Correlación entre los factores } \\
\hline Factor 1 & - & & & \\
\hline Factor 2 & .14 & - & & \\
\hline Factor 3 & .46 & .49 & - & \\
\hline
\end{tabular}

Nota: F1=Búsqueda de apoyo social, F2=Solución técnica evasiva y F3=Solución técnica proactiva; $h^{2}=$ comunalidad.

Análisis factorial confirmatorio. Se realizó el análisis factorial confirmatorio tomando la SM2 y los 12 ítems que resultaron del análisis previo. En este análisis se identificó un ítem que no ajusta en el modelo (Cambio contraseña, nombre de usuario, cuenta de correo), por lo que no se incluyó en análisis posteriores.

La figura 1 presenta el modelo final resultante, que incluye 11 ítems agrupados en tres dimensiones de la escala ACES: a) Búsqueda de apoyo social (5 ítems), b) Solución técnica evasiva (3 ítems) y c) Solución técnica proactiva (3 ítems). Los índices de bondad de ajuste fueron: $X^{2}=59.06, g l=40, p=.026$, SRMR $=.07$, GFI $=.97, \mathrm{CFI}=.99$, RMSEA $=.03$ IC 90 [.01-.05]; los cuales muestran una adecuada bondad de ajuste, con un valor de $X^{2}$ adecuado al tamaño de la muestra y a la normalidad de los datos, por lo que no resultó significativo. Lo anterior representa un modelo adecuado para la medición del constructo (Cea, 2004). Además, se aprecia que las cargas factoriales de cada ítem son significativas. El valor de la covarianza entre los factores sugiere que miden distintos tipos de afrontamiento. 


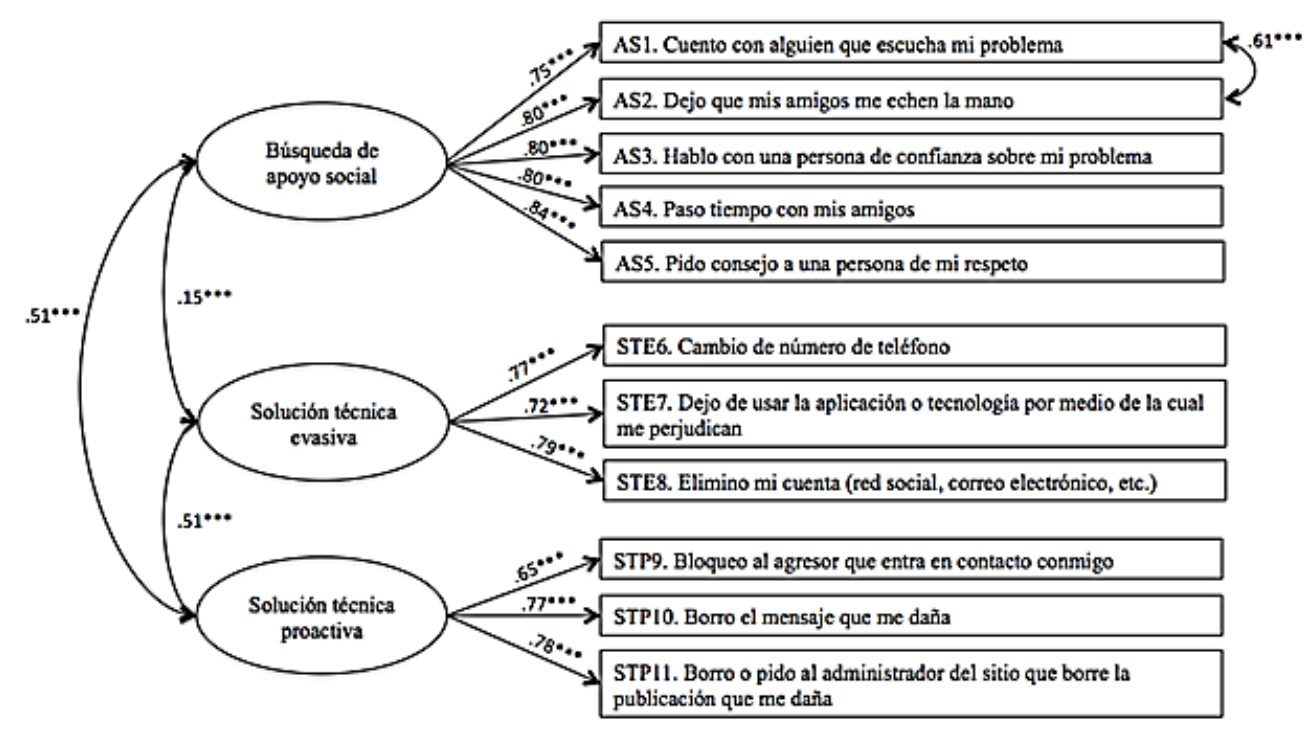

Nota. Se reportan los coeficientes estandarizados

Figura 1. Resultados del análisis factorial confirmatorio de la escala ACES (*** $p<.000)$

Análisis de fiabilidad. Para determinar la fiabilidad de cada factor y de la escala total se emplean tres coeficientes, en la tabla III se muestran los resultados. Los valores del Alfa de Cronbach, Omega de McDonald $(\Omega)$ y Varianzas Medias Extractadas (VME) resultan en niveles aceptables de fiabilidad tanto en las dimensiones como a nivel global (Furr y Bacharach, 2014; Zumbo, Gaderman y Zeissen, 2007).

Tabla III. Fiabilidad de la escala ACES y sus factores

\begin{tabular}{l|c|c|c|c}
\hline \multirow{2}{*}{ Fiabilidad } & \multicolumn{3}{|c|}{ Factores } & \multirow{2}{*}{ Global } \\
\cline { 2 - 4 } & $\begin{array}{c}\text { Búsqueda } \\
\text { de apoyo social }\end{array}$ & $\begin{array}{c}\text { Solución } \\
\text { técnica evasiva }\end{array}$ & $\begin{array}{c}\text { Solución } \\
\text { técnica proactiva }\end{array}$ & \\
\hline Alfa de Cronbach & .91 & .80 & .78 & .85 \\
Omega de McDonald & .85 & .70 & .66 & .91 \\
Varianzas Medias Extractadas (VME) & .64 & .58 & .54 & .54 \\
\hline
\end{tabular}

\section{Discusión y conclusiones}

Este estudio plantea como objetivo el desarrollo de una escala para medir el afrontamiento frente a ciberagresiones por parte de estudiantes universitarios de una universidad al sur de Sonora. Los resultados muestran evidencia de un instrumento válido y confiable para la medición del constructo, conformado por tres dimensiones: búsqueda de apoyo social, solución técnica evasiva y solución técnica proactiva.

En términos metodológicos, los resultados encontrados son un primer acercamiento que demuestra la validez y fiabilidad de la escala propuesta, y representan una contribución práctica y teórica al estudio del afrontamiento frente a ciberagresiones. Desde el punto de vista práctico se aporta un instrumento con propiedades psicométricas adecuadas para la medición del constructo con estudiantes de educación superior en México. En cuanto al aporte teórico, los hallazgos encontrados permiten delimitar el tipo de afrontamiento que los estudiantes universitarios practican cuando se encuentran frente a situaciones específicas de ciberbullying. El análisis de la información permite aportar una subclasificación de soluciones técnicas (solución técnica evasiva y solución técnica proactiva); así como validar la dimensión 
de búsqueda de apoyo con población mexicana.

Por otra parte, la clasificación de ítems propuestos en la figura 1 permite que profesores, padres de familia y comunidad educativa en general puedan establecer estrategias para atender y prevenir los efectos que se logran en las víctimas (Parris et al., 2012).

Por último, entre las limitaciones del estudio se reconoce el tamaño de la muestra, que, aunque es suficiente para los análisis realizados es limitada para llevar a cabo otros análisis de validez, tales como la invarianza, por lo que se recomienda ampliarla en futuros estudios e incluso considerar a estudiantes universitarios de todo el territorio mexicano y de otras áreas del conocimiento; se recomienda también incluir elementos cualitativos que permitan a los participantes expresar otras posibles acciones de afrontamiento.

\section{Referencias}

Aricak, T., Siyahhan, S., Uzunhasanoglu, A., Saribeyoglu, S., Ciplak, S., Yilmaz, N. y Memmedov, C. (2008). Cyberbullying among turkish adolescents. CyberPsychology \& Behavior, 11(3), 253-261.

doi:10.1089/cpb.2007.0016

Belsey, B. (2005). Cyberbullying: an emerging threat to the always on generation. Recuperado de http://www.cyberbullying.ca

Brown, T. (2015). Confirmatory factor analysis for applied research (2a. ed.). Nueva York: The Guilford Press.

Buelga, S., Cava, M. y Musitu, G. (2010). Cyberbullying: victimización entre adolescentes a través del teléfono móvil y de internet. Psicothema, 22(4), 784-789. Recuperado de

http://www.psicothema.com/resumen.asp?id=3802

Byrne, B. (2010). Structural equation modeling with Amos. Basic concepts, applications, and programming (2a. ed.). NuevaYork: Routledge Taylor \& Francis Group.

Canessa, B. (2002). Adaptación psicométrica de las escalas de afrontamiento para adolescentes de Frydenberg y Lewis en un grupo de escolares de Lima metropolitana. Persona, 5, 191-233. Recuperado de https://revistas.ulima.edu.pe/index.php/Persona/article/view/876/826

Cano, F., Rodríguez, L. y García, J. (2007). Adaptación española del inventario de estrategias de afrontamiento. Actas Españolas de Psiquiatría, 35(1), 29-39.

Causey, D. y Dubow, E. (1992). Development of a self-report coping measure for elementary school children. Journal of Clinical Child Psychology, 21(1), 47-59. doi: 10.1207/s15374424jccp2101_8

Cea, M. (2004). Análisis multivariable. Teoría y práctica en la investigación social. Madrid: Síntesis.

Chapell, M., Hasselman, S., Kitchin, T., Lomon, S., Maclver, K. y Sarullo, P. (2006). Bullying in elementary school, high school, and college. Adolescence, 41(164), 633-648. Recuperado de

https://www.ncbi.nlm.nih.gov/pubmed/17240771

De Vellis, R. (2012). Scale development. Theory and Aplications (3a. ed.). Los Ángeles: Sage.

Fernández, M. D. y Díaz, M. (2001). Relación entre estrategias de afrontamiento, síndromes clínicos y transtornos de personalidad en pacientes esquizofrénicos crónicos. Revista de Psicopatología y Psicología Clínica, 6(2), 129-135. doi:10.5944/rppc.vol.6.num.2.2001.3910 
Frydenberg, E. y Lewis, R. (1994). Coping with different concerns: consistency and variation in coping strategies used by adolescents. Australian Psychologist, 29(1), 45-48. doi: 10.1080/00050069408257320

Frydenberg, E. y Lewis, R. (1999). Things don't better just because you're older: a case for facilitating reflection. British Journal of Educational Psychology, 69(1), 81-94. doi: 10.1348/000709999157581

Furr, M. y Bacharach, V. (2014). Psychometrics. An introduction (2a. ed.). Nueva York: Sage.

Garaigordobil, M. (enero-marzo, 2011). Bullying y cyberbullying: conceptualización, prevalencia y evaluación. Formación Continuada a Distancia, duodécima edición, 3-13. Recuperado de

https://docplayer.es/9265508-Bullying-y-cyberbullying-conceptualizacion-prevalencia-y-evaluacion.html

García-Maldonado, G., Joffre-Velázquez, V., Martínez-Salazar, G. y Llanes-Castillo, A. (2011). Ciberbullying: forma virtual de intimidación escolar. Revista Colombiana de Psiquiatría, 40(1), 115-130.

doi:10.1016/S0034-7450(14)60108-6

Hoff, D. y Mitchell, S. (2009). Cyberbullying: causes, effects, and remedies. Journal of Educational Administration, 47(5), 652-665. doi:10.1108/09578230910981107

Holfeld, B. y Leadbeater, B. (2014). The nature and frequency of cyber bullying behaviors and victimization experiences in young canadian children. Canadian Journal of School Psychology, 30(2), 116135. doi:10.1177/0829573514556853

Kristensen, S. y Smith, P. (2003). The use of coping strategies by Danish children classed as bullies, victims, bully/victims, and not involved, in response to different (hypothetical) types of bullying. Scandinavian Journal of Psychology, 44(3), 479-488. doi:10.1046/j.1467-9450.2003.00369.x

Lazarus, R. y Folkman, S. (1984). Stress, appraisal and coping. Nueva York: Springer.

Lazarus, R. y Folkman, S. (1986). Estrés y procesos cognitivos. Barcelona: Martínez Roca.

Lazarus, R. y Folkman, S. (1987). Transactional theory and research on emotions and coping. European Journal of Personality, 1(3), 141-169. doi:10.1002/рег.2410010304

Lazarus, R. (2006). Emotions and interpersonal relationships: toward a person-centered conceptualization o emotions and coping. Journal of Personality, 74(1), 9.46. doi:10.1111/j.1467-6494.2005.00368.x

Li, Q. (2008). A cross-cultural comparison of adolescents' experience related to cyberbullying. Educational Research, 50(3), 223-234. doi:10.1080/00131880802309333

Lin, N., Dean, A. y Ensel, W. (1981). Social support scales: a methodological note. Schizophrenia Bulletin, 7(1), 73-89. Recuperado de https://www.ncbi.nlm.nih.gov/pubmed/7233114

Livingstone, S., Haddon, L., Gözig, A. y Ólafsson, K. (2011). Risks and safety on the Internet: the perspective of european children: full findings and policy implications from the EU kids online survey of 9-16 year olds and their parents in 25 countries. Londres: LSE.

Machackova, H., Cerna, A., Sevcikova, A., Dedkova, L. y Danebach, K. (2013). Effectiveness of coping strategies for victims of cyberbullying. Cyberpsychology: Journal of Psychosocial Research on Cyberspace, 7(3). doi:10.5817/CP2013-3-5

Machmutow, K., Perren, S., Sticca, F. y Alsaker, F. (2012). Peer victimisation and depressive symptoms: can specific coping strategies buffer the negative impact of cybervictimisation? Emotional and Behavior Difficulties, 17(3-4), 403-420. doi:10.1080/13632752.2012.704310 
Moral, J., López, F., Díaz, R. y Cienfuegos, Y. (2011). Diferencias de género en afrontamiento y violencia en la pareja. Revista CES Psicología, 4(2), 29-46. Recuperado de http://revistas.ces.edu.co/index.php/psicologia/article/view/1502

Nacimiento, L. y Mora-Merchán, J. (2014). El uso de estrategias de afrontamiento y habilidades metacognitivas ante situaciones de bullying y ciberbullying. European Journal of Education and Psychology, 7(2), 121-129. doi:10.30552/ejep.v7i2.104

Nava, C., Ollua, P., Vega, C. y Soria, R. (2010). Inventario de estrategias de afrontamiento: una replicación. Psicología y Salud, 20(2), 213-220. Recuperado de http://www.uv.mx/psicysalud/psicysalud-20-2/202/Carlos-Nava-Quiroz.pdf

Nocentini, A., Calmaestra, J., Schultze-Krumbholz, A., Scheithauer, H., Ortega, R. y Menesini, E. (2010). Cyberbullying: labels, behaviours and definition in three european countries. Australian Journal of Guidance \& Counselling, 20(2), 129-142. doi:10.1375/ajgc.20.2.129

Oliveros, M., Amemiya, I., Condorimay, Y., Oliveros, R., Barrientos, A. y Rivas, B. (2012). Ciberbullying: nueva tecnología electrónica al servicio del acoso escolar en alumnos de dos distritos de Lima, Perú. Anales de la Facultad de Medicina, 73(1), 13-18. doi:10.15381/anales.v73i1.804

Olweus, D. (2012). Cyberbullying: an overrated phenomenon? European Journal of Developmental Psychology, 9(5), 520-538. doi:10.1080/17405629.2012.682358

Parris, L., Varjas, K., Meyers, J. y Cutts, H. (2012). High school students' perceptions of coping with cyberbullying. Youth \& Society, 44(2), 284-306. doi:10.1177/0044118X11398881

Paul, S., Smith, P. y Blumberg, H. (2012). Comparing student perceptions of coping strategies and school interventions in managing bullying and cyberbullying incidents. Pastoral Care in Education, 30(2), 127-146. doi:10.1080/02643944.2012.679957

Perren, S., Corcoran, L., Cowie, H., Dehue, F., García, D., McGuckin, C... Völlink, T. (2012). Tackling cyberbullying: review of empirical evidence regarding successful responses by student, parents, and schools. International Journal of Conflict and Violence, 6(2), 283-293. doi:10.4119/UNIBI/ijcv.244

Perren, S., Dooley, J., Shaw, T. y Cross, D. (2010). Bullying in school and cyberspace: associations with depressive symptoms in swiss and australian adolescents. Child and Adolescent Psychiatry and Mental Health, 4(28), 1-10. doi:10.1186/1753-2000-4-28

Price, M. y Dalgleish, J. (2010). Cyberbullying. Experiences, impacts and coping strategies as described by Australian young people. Youth Studies Australia, 29(2), 51-59.

Puc, E. y Rodríguez, A. (2015). Cyberbullying: una exploración descriptiva en estudiantes universitarios. Lenguas en contexto (ed. esp.), 38-46. Recuperado de

http://www.facultaddelenguas.com/lencontexto/?seccion=revista\&idrevista=20

Sahin, M., Aydin, B. y Sari, S. (2012). Cyber bullying, cyber victimization and psychological symptoms: a study in adolescents. Faculty of Education Journal, 41(1), 53-59. Recuperado de http://dergipark.gov.tr/download/article-file/46475

Schenk, A. y Fremouw, W. (2012). Prevalence, psychological impact, and coping of cyberbully victims among college students. Journal of School Violence, 11(1), 21-37. doi:10.1080/15388220.2011.630310

Sevcíkova, A., Machackova, H., Wright, M., Debková, L. y Cerna, A. (2015). Social support seeking in relation to parental attachment and peer relationships among victims of cyberbullying. Journal of Psychologists and Counsellors in Schools, 25(2), 170-182. doi:10.1017/jgc.2015.1 
Sléglová, V. y Cerná, A. (2011). Cyberbullying in adolescent victims: perception and coping. Cyberpsychology: Journal of Psychosocial Research on Cyberspace, 5(2), 1-16. Recuperado de http://cyberpsychology.eu/view.php?cisloclanku=2011121901\&article=4

Slonje, R. y Smith, P. (2008). Cyberbullying: another main type of bullying? Scandinavian Journal of Psychology, 49(2), 147-154. doi:10.1111/j.1467-9450.2007.00611.x

Slonje, R., Smith, P. y Frisén, A. (2013). The nature of cyberbullying, and strategies for prevention. Computers in Human Behavior, 29, 26-32. doi:10.1016/j.chb.2012.05.024

Smith, P., Mahdavi, J., Carvalho, M., Fisher, S., Russell, S. y Tippett, N. (2008). Cyberbullying: its nature and impact in secondary school pupils. Journal of Child Psychology and Psychiatry, 49(4), 376-385.

doi:10.1111/j.1469-7610.2007.01846.x

Tokunaga, R. (2010). Following you home from school: a critical review and synthesis of research on cyberbullying victimization. Computers in Human Behavior, 26(3), 277-287. doi:10.1016/j.chb.2009.11.014

Torres-Acuña, G., Rivera, C., Navarro, Y. y Sandoval, V. (noviembre, 2016). Afrontamiento empleado por estudiantes de bachillerato con y sin reportes de cibervictimización. Ponencia presentada en el 8o. Congreso Internacional de Educación. Sonora, México.

Valdés, A., Carlos, W., Tanori, J. y Wendlandt, T. (2014). Differences in types and technological means by which mexican high schools students perform cyberbullying: its relationship with traditional bullying. Journal of Educational and Developmental Psychology, 4(1), 105-113. doi:10.5539/jedp.v4n1p105

Varela, J., Pérez, C., Schwaderer, H., Astudillo, J. y Lecannelier, F. (2014). Caracterización de cyberbullying en el gran Santiago de Chile. Psicologia Escolar e Educacional, 18(2), 347-354.

doi:10.1590/2175-3539/2014/0182794

Vega-López, M., González-Pérez, G. y Quintero-Vega, P. (2013). Ciberacoso: victimización de alumnos en escuelas secundarias públicas de Tlaquepaque, Jalisco, México. Revista de Educación y Desarrollo, 25, 1320. Recuperado de http://www.cucs.udg.mx/revistas/edu desarrollo/anteriores/25/025 Vega.pdf

Völlink, T., Bolman, C., Dehue, F. y Jacobs, N. (2013). Coping with cyberbullying: differences between victims, bully-victims and children not involved in bullying. Journal of Community \& Applied Social Psycholy, 23(1), 7-24. doi:10.1002/casp.2142

Wachs, S., Wolf, K. y Pan, C. (2012). Cybergrooming: risk factors, coping strategies and associations with cyberbullying. Psicothema, 24(4), 628-633. Recuperado de

http://www.psicothema.com/psicothema.asp?id=4064

Zumbo, B., Gaderman, A. y Zeissen, C. (2007). Ordinal version of coefficients Alpha and Theta for Likert rating scales. Journal of Modern Applied Statistical Methods, 6(1), 21-29. doi:10.22237/jmasm/1177992180 